\title{
Delayed switching applied to memristor neural networks
}

\author{
Frank Z. Wang, ${ }^{1, a)}$ Na Helian, ${ }^{2}$ Yike Guo, ${ }^{3}$ Sining Wu, ${ }^{1}$ Xiao Yang,,${ }^{1}$ Guan Lim, ${ }^{1}$ \\ and Md Mamunur Rashid ${ }^{4}$ \\ ${ }^{1}$ Future Computing Group, School of Computing, University of Kent, United Kingdom \\ ${ }^{2}$ School of Computer Science, University of Hertfordshire, United Kingdom \\ ${ }^{3}$ Department of Computing, Imperial College, United Kingdom \\ ${ }^{4}$ CERN, Geneva, Switzerland
}

(Presented 3 November 2011; received 28 September 2011; accepted 26 October 2011; published online $\mathrm{xx} \mathrm{xx} \mathrm{xxxx}$ )

\begin{abstract}
Magnetic flux and electric charge are linked in a memristor. We reported recently that a memristor has a peculiar effect in which the switching takes place with a time delay because a memristor possesses a certain inertia. This effect was named the "delayed switching effect." In this work, we elaborate on the importance of delayed switching in a brain-like computer using memristor neural networks. The effect is used to control the switching of a memristor synapse between two neurons that fire together (the Hebbian rule). A theoretical formula is found, and the design is verified by a simulation. We have also built an experimental setup consisting of electronic memristive synapses and electronic neurons. (C) 2012 American Institute of Physics. [doi:10.1063/1.3672409]
\end{abstract}

\section{INTRODUCTION}

Since the first computer was built, scientists have been fascinated with the idea of a computer that works similarly as the human brain. However, all previous efforts at building brain-like computers failed because it took about the same silicon area to emulate a CMOS synapse as was needed to emulate a neuron. In a typical human brain, there are $10^{11}$ neurons and $10^{14}$ synapses (on average, each neuron is connected to other neurons through about 20000 synapses). Any realistic implementation of a synapse should ideally be at least four orders of magnitude smaller than that required to build a neuron. Although the implementation of a neuron is relatively easier, an electronic synapse is not so straightforward to make for the above-stated reason.

The invention of the memristor ${ }^{1}$ provides a new way to implement synapses. A memristor is a simple 2-terminal element, which means a vast number of memristors could be integrated together with other CMOS elements in a single chip. $\mathrm{A} \mathrm{LaAlO}_{3} / \mathrm{SrTiO}_{3}$ junction presents a uni-polar pinched hysteresis loop and also provides the potential for a memristor to be scaled down to half a nanometer. ${ }^{2}$ Memristors are passive and non-volatile and consume much less power.

Naturally, the freezing memory property by which a memristor stores resistance value makes memristors suitable for use as synapses. As shown in Fig. 1(b), the switching from high resistance $\left(R_{\text {off }}\right)$ to low resistance $\left(R_{\text {on }}\right)$ takes place with a time delay $T_{d}$ after the application of an input voltage. In a memristor neural network, a square-wave signal is equivalent, in terms of switching a memristor synapse, to a sequence of spikes with the same net area as the observation region bounded by the graph of the signal and the time axis (Fig. 1(c)). This is because charge is the time integral of current, and the mem-resistance is normally a function of charge.

\footnotetext{
a) Author to whom correspondence should be addressed. Electronic mail: frankwang@ieee.org.
}

The resistance of a memristor depends on the complete 50 past history of the current, i.e., the time integral of the cur- 51 rent from $\tau=-\infty$ to $\tau=t$. As mentioned above, the current 52 (voltage) is a sequence of spikes with a frequency $f$ and a 53 (equal) spike width $T_{w}$. Therefore,

$$
q(t)=\int_{-\infty}^{+t} i(\tau) d \tau=\int_{-\infty}^{+t} \frac{V d \tau}{R}=\frac{V}{R} \cdot T_{w} \cdot f \cdot t .
$$

At the transition where $t=T_{d 1,2}$, we have $q\left(T_{d 1,2}\right)=Q_{1,2}$ and 55 $\varphi\left(T_{d 1,2}\right)=\Phi_{1,2}$. Therefore,

$$
\begin{gathered}
Q_{1,2}=\frac{V}{\frac{\phi_{1,2}}{Q_{1,2}} \cdot T_{W} \cdot f \cdot T_{d 1,2}} \\
T_{d 1,2}=\frac{\phi_{1,2}}{V \cdot T_{W} \cdot f}, \quad T_{d 1}=\frac{\phi_{1}}{V \cdot T_{W} \cdot f}, \quad T_{d 2}=\frac{\phi_{2}}{V \cdot T_{W} \cdot f} .
\end{gathered}
$$

Equation (3) clearly demonstrates that $T_{d}$ decreases with an increased spike amplitude $V$, an increased spike width $T_{W}$, or an increased spike frequency $f$. If the input voltage is removed before the switching takes place, i.e., the width $T$ of the input voltage pulse is smaller than $T_{d} \approx T_{d 1} \approx T_{d 2}$, the memristor remains unaltered. Therefore, in order to switch a memristor, $T$ should be chosen in such a way that $T>T_{d}$.

\section{DELAYED SWITCH IN MEMRISTOR NEURAL NETWORKS}

As shown in Fig. 2, we consider a simplified neural net- 66 work comprising three neurons (N1, N2, and N3) coupled by 67 two memristive synapses (S1 and S2). This network can 68 perform the Pavlovian experiment on conditioned reflex. The 69 first input neuron (presumably located in the visual cortex) 70 activates under a specific visual event, such as "sight of 71 food," and the second input neuron (presumably located in 72 

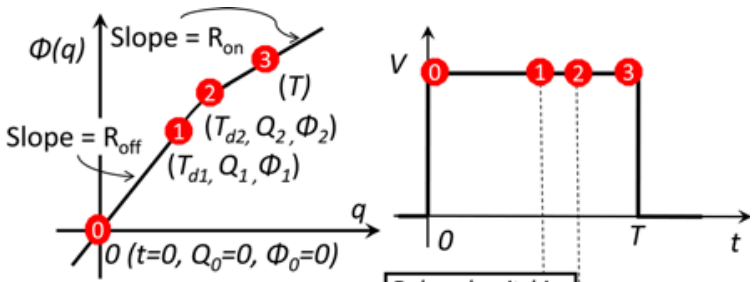

(a) $\phi$-q curve;

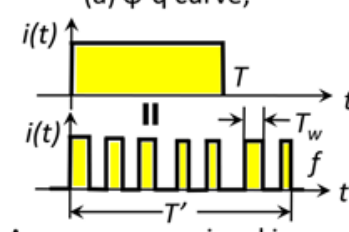

(c) A square-wave signal is

equivalent to a sequence of spike with the same total area over $T$.

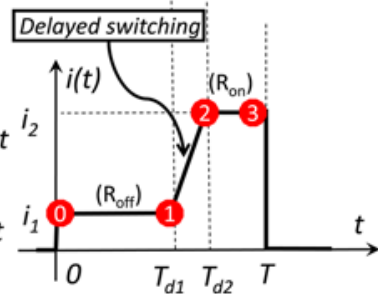

(b) "Full Voltage" excitation;

FIG. 1. (Color online) Memristor's delayed switching effect (Refs. 3 and 4): the switching from one resistance state to another due to an input voltage pulse takes place with a time delay. The effect also applies to a sequence of spikes, well used in neural networks.

the auditory cortex) activates under an external auditory event, such as "sound of bell." Depending on previous training, each of these events can trigger "salivation" (firing of the third output neuron). If, at a certain moment in time, only the sight of food leads to salivation, and subsequently the circuit is subjected to both input events, then, after a sufficient number of simultaneous input events, the circuit starts associating the sound of a bell with the sight of food, and eventually it begins to salivate upon the activation of the sound only. This process of learning is a realization of the famous Hebbian rule. ${ }^{5}$

A biological neuron behaves like an analog-to-digital converter. When a neuron receives a receptor potential exceeding a threshold value, it starts emitting both forward (along the output terminal of the neuron) and backward (along the input of the neuron) action spikes; the amplitude of these spikes is constant, but their frequency depends on the stimulus strength.

A biological synapse is a connection that permits a neuron to pass an electrical signal to another neuron. Our memristor synapse is modeled in Fig. 1(a). As shown in Fig. 2, the synapse $\mathrm{S} 1$ receives a voltage determined by the output of the neuron N1 at its front (forward-propagating spikes) and the input of another neuron N3 at its back (back-

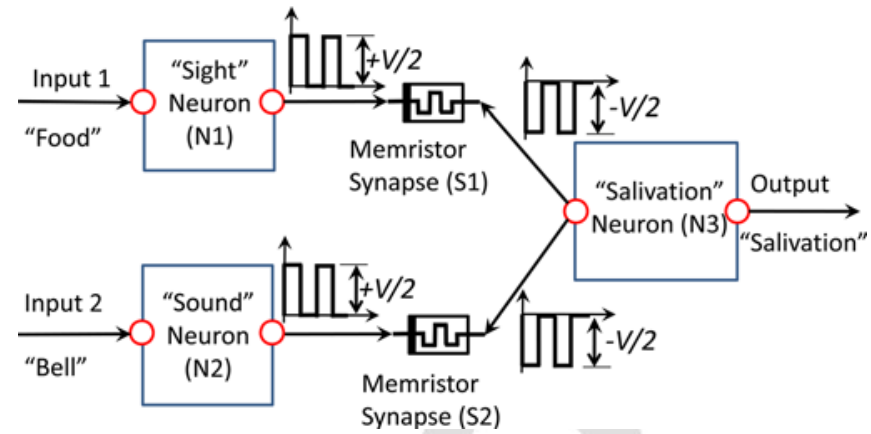

FIG. 2. (Color online) A neural network using memristors as synapses. When a neuron fires, it starts emitting a forward spike, $+V / 2$, and a backward spike, $-V / 2$. The strengths of memristor synapses can be modified when these two spikes overlap.

propagating spikes). If the pre-synaptic and post-synaptic 96 spikes overlap, a positive "half voltage" $(+v / 2)$ and a nega- 97 tive "half voltage" $(-v / 2)$ generate a "full voltage" drop $(v) 98$ across the synapse.

The simulation results are shown in Fig. 3. The width of 100 each pulse (spike) is set at 4 time units, and the memristor 101 synapse S2 remains unaltered (still disconnected). The simu- 102 lation period is 6600 time units.

In the "probing" phase ("food" only or "bell" only in 104 Fig. 3), the salivation neuron fires only when a stimulus 105 signal is applied to the sight neuron, as S1 is connected and 106 $\mathrm{S} 2$ is disconnected.

In the "learning" phase (Fig. 3), stimulus voltages are 108 applied simultaneously to both input neurons ("sight" and 109 "sound"), thus generating a sequence of spikes. The spikes 110 from different neurons are uncorrelated, but sometimes they 111 do overlap, owing to a random component in the spike sepa- 112 ration. During this phase, in some moments of time, back- 113 propagating spikes from the salivation neuron (due to excita- 114 tion from the sight neuron) overlap with forward propagating 115 spikes from the sound neuron, causing a full voltage across 116 the second memristor synapse S2. As this voltage exceeds 117 the memristor threshold, S2 changes its state and switches 118 into a low resistance state (connection). It is important to 119 note that this change is possible when both stimuli are 120 applied together (in other words, they correlate). As a result, 121 an association between input stimuli develops, and the 122

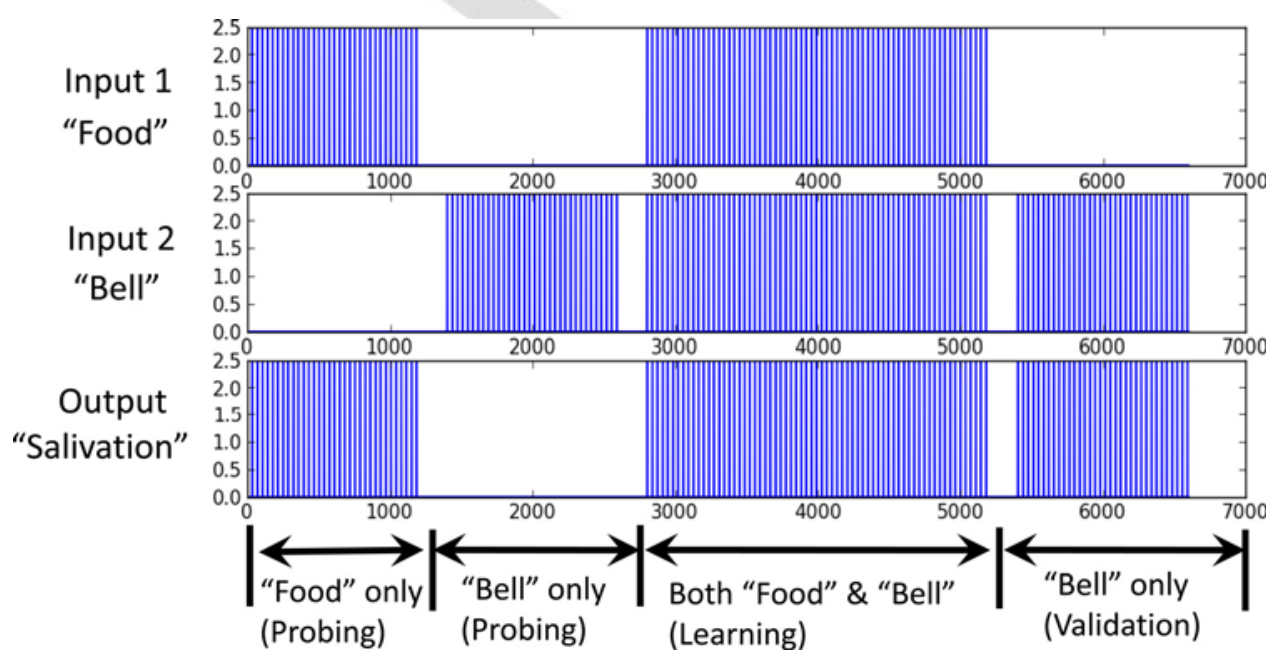

FIG. 3. (Color online) Simulation demonstration of the memristor neural network in Fig. 2. 


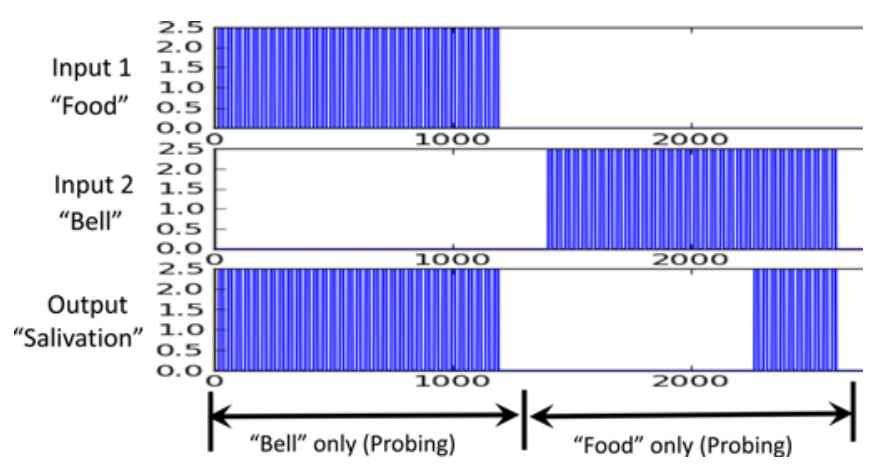

FIG. 4. (Color online) If each spike is too wide or the spike sequence is too long, the "salivation" will be triggered by mistake when only the "sound" neuron fires.

network "learns" to associate the sight neuron with the sound neuron.

Our measurements during the second probing phase ("validation" in Fig. 3) clearly demonstrate the developed association. In this phase, any type of stimulus, whether from the sight neuron or from the sound neuron, results in the firing of the salivation neuron.

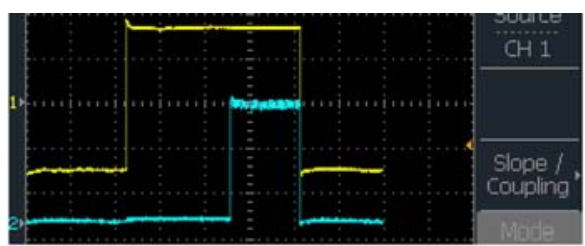

(a) A "Full Voltage" causes a delayed switching;

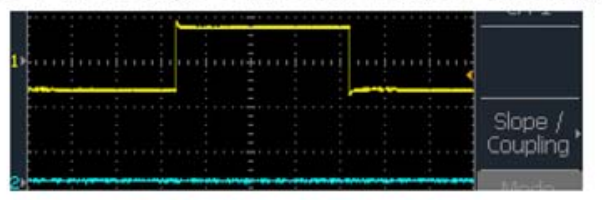

(b) A "Half Voltage" causes no switching;

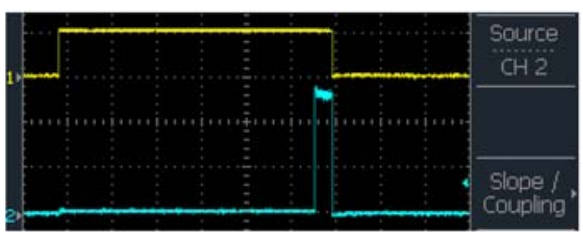

(c) A long "Half Voltage" still causes an unwanted switching.

FIG. 5. (Color online) Measured waveform on an experimental setup consisting of electronic memristive synapses and electronic neurons. The "full voltage" is $5 \mathrm{~V}$, and the "half voltage" is $2.5 \mathrm{~V}$.

If the width of each spike is increased to 16 time units 130 (with the same frequency), the integral (charge) rises quickly 131 and the memristor time delay (Eq. (3)) is overtaken; as a 132 result, S2 will change its state even under a half voltage 133 (only the sound neuron fires), as shown in Fig. 4. Obviously 134 this is a mis-operation in which the sound neuron is con- 135 nected with the salivation neuron by mistake, violating the 136 Hebbian rule ("neurons that fire together, wire together"). 137

\section{CIRCUIT EXPERIMENTS}

Based on Chua's "circuit-model,", we have built an ex- 139 perimental setup consisting of electronic memristive synap- 140 ses and electronic neurons. ${ }^{3-5}$ The electronic memristor 141 synapse can be tuned to represent the functions found in bio- 142 logical neural cells. ${ }^{5} 143$

The waveforms are measured with a 16 to $25 \mathrm{~Hz}$ square- 144 wave input signal, a low resistance of $625 \Omega$, and a high 145 resistance of $10 \mathrm{k} \Omega$. As shown in Fig. 5, the application of 146 the delayed switching in a neural network has been achieved. 147

\section{CONCLUSION}

148

A memristor mimics the synapses between the neurons 149 in the brain in terms of being plastic according to the dynam- 150 ical history of the system. According to Eq. (3), the sequence 151 length, sequence frequency, and spike width need to be care- 152 fully controlled in such a way that the memristor synapse 153 time delay point is not be overtaken while only one neuron 154 fires.

\section{ACKNOWLEDGMENTS}

This research was conducted with partial support 157 from UK Government EPSRC Grant (EP/E064930/1). The 158 authors wish to thank Professor Leon Chua (University of 159 California) for his helpful comments and suggestions while 160 he was a Leverhulme Trust Visiting Professor in the UK 161 over the past year.

${ }^{1}$ L. Chua, IEEE Trans. Circuit Theory 18(5), (1971).

T. Fix, J. L. MacManus-Driscoll, and M. G. Blamire, Appl. Phys. Lett. 94, 165 (2009).

${ }^{3}$ F. Z. Wang et al., IEEE Electron Device Lett. 31(7), (2010).

${ }^{4} \mathrm{~F}$. Wang et al., paper presented at the Magnetism and Magnetic Materials 168 Conference, 2010.

${ }^{5}$ Y. Pershin and M. Di Ventra, "Experimental demonstration of associative 170 memory with memristive neural networks," Nature Precedings, available at 171 http://precedings.nature.com/documents/3258/version/1. 